\title{
RELIABILITY ASSESSMENT OF HIGH-SPEED TRAIN BEARING BASED ON RANDOM PERFORMANCE DEGRADATION IN MINIMUM SAMPLE
}

\author{
Dexin $\mathrm{ZHU}^{1}$, Nan CUI ${ }^{2}$ \\ 1,2Beifang University of Nationalities, Yinchuan, 750021, China \\ Corresponding author: Dexin ZHU \\ Email: zhudexinscience@163.com
}

\begin{abstract}
Considering that it is difficult to make the large sample test and obtain the failure data in the limited test time for high-speed train bearing (HSTB) test, the random performance degradation process model based on the degradation data of bearing relative temperature is used to obtain the reliability function of bearing life with the characteristics of the slow degradation process and relatively stable degradation path. The degradation path model is reasonably given through viewing the unknown coefficients of the model as random variables. According to the analysis to a bearing testing data, the reliability assessment of the bearing life is finally completed, while a guiding role is provided for the reliability assessment problem of HSTB.
\end{abstract}

Keywords: HSTB; Wiener Process; Performance Degradation; Bootstrap Estimation; Reliability Assessment

\section{Introduction}

At present, it is difficult to make the large sample test and obtain the failure data in the limited test time for the high-speed train bearing (HSTB) test, because the bearing is very expensive, the requirements of accuracy and reliability are also quite high. Therefore, it is worth studying how to assess the reliability life of the new developed HSTB using the limited test data obtained in the condition of the small samples even minimum and zero-failure.

The author has proposed a traditional Bayes assessment method [1]. However, the existing question is how to determine the prior distribution when using the Bayes method. If the prior distribution is very reasonable, the result would be more realistic, and the practical experience could be successfully used [2]. For the reliability assessment with a small sample and zero-failure data, the performance degradation data are also a useful information [3].

E.g. Peng [4] uses a Wiener-process-based degradation model with a recursive filter algorithm to solve the problem of estimating the remaining useful life from the observed degradation data. Tseng [5] and Tang [6] et al. have also adopted Wiener process to model. Wang [7] has studied the two kinds of the lifetime prediction based on Gamma processes. Pan [8] proposes a bivariate BirnbaumSaunders distribution and its marginal distributions to approximate the reliability function [9].
Tsai [10] uses the gamma process to provide information about the reliability of highly reliable products that are not likely to fail within a reasonable period under traditional life tests, or even accelerated life tests.

This paper aims to develop a new reliability analysis method based on the random performance degradation model. We expect to provide an effective tool of reliability assessment for HSTB in the minimum samples and zero-failure.

\section{Performance Degradation Model}

\subsection{Model Assumption}

The degradation model satisfies the following assumptions:

I. Product failure is caused by degradation, and the degradation failure shows that the change trend of a certain performance parameter is monotonous over time. Let $\mathrm{Z}(\mathrm{t})$ denote the performance parameter value at time $t$. The measured data, written as $X(t)$, corresponding to $\mathrm{Z}(\mathrm{t})$, are called the degraded. And the measured error is written as $\varepsilon(t)$. Then we have $\mathrm{Z}(\mathrm{t})=\mathrm{X}(\mathrm{t})+\varepsilon(\mathrm{t})$. In this paper, we wouldn't consider the measured error, i.e. $\mathrm{Z}(\mathrm{t})=\mathrm{X}(\mathrm{t})$.

II. The tested samples are randomly selected from the general, and the test condition and the measure errors of all products are same.

III. The observed values of performance parameter obey the independent and identical distribution at 
any time, whether it is continuum damage or discrete. And the measure is nondestructive.

\subsection{Degradation Model}

Supposing there are $\mathrm{n}$ samples measured in the performance degradation test. The $m$ times measures of test data are respectively made at time $t 1, t 2, \ldots, t m$. Then the performance degradation data are obtained as follows

$\mathrm{X} 1(\mathrm{t} 1), \mathrm{X} 1(\mathrm{t} 2), \ldots, \mathrm{X} 1(\mathrm{tm})$

$\mathrm{X} 2(\mathrm{t} 1), \mathrm{X} 2(\mathrm{t} 2), \ldots, \mathrm{X} 2(\mathrm{tm})$

…….....

$\mathrm{Xn}(\mathrm{t} 1), \mathrm{Xn}(\mathrm{t} 2), \ldots, \mathrm{Xn}(\mathrm{tm})$.

Where $\mathrm{Xi}(\mathrm{tj})$ is the performance degradation value for ith sample at time $t j, i=1,2, \ldots, n, j=1,2, \ldots, m$.
According to the assumption III, we know that X1(ti), $\mathrm{X} 2(\mathrm{ti}), \ldots, \mathrm{Xn}(\mathrm{ti})$ obey the independent and identical distribution.

To estimate the sample reliability, we process for the obtained data. The detailed procedure as follows:

1) Collecting the degradation data, making the statistical analysis, removing the anomalous data, and judging which distribution is coincident with the performance degradation value at every moment.

2) Solving the average and variance of performance degradation value at all times and finally computing the reliability of the tested bearings.

As shown in Fig.1, it is the tendency chart of the tested bearings about relative temperature, which is relative to the ambient temperature.

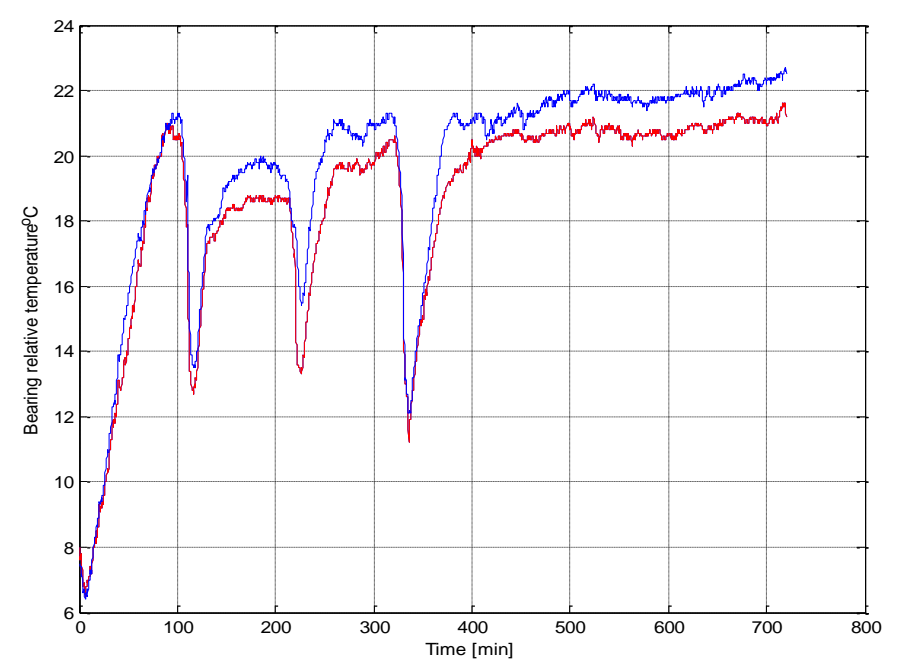

Figure 1: The changed tendency of bearings relative temperature

From Fig. 1, although we can see three times sudden change of curve, it is caused by the suspension of the testing machine. Because the lubrication pressure is under the lower limit of the set value during the test, the bearings relative temperature rises slowly with time.

In view of the above features about the bearing relative temperature, we use the Wiener process to model, its mathematical model can be expressed as follows:

$$
\mathrm{X}(\mathrm{t} ; \mathrm{v}, \delta 2)=\mathrm{b}+\mathrm{vt}+\delta \mathrm{W}(\mathrm{t})
$$

Where $\mathrm{X}(\mathrm{t} ; \mathrm{v}, \delta 2)$ is the relative temperature of bearing at time $t, b$ is the initial value of bearing relative temperature; $\mathrm{v}$ and $\delta$ are the unknown parameters;

$\mathrm{W}(\mathrm{t})$ is the standard Wiener process, with $\mathrm{E}[\mathrm{W}(\mathrm{t})]=0$, $\mathrm{E}[\mathrm{W}(\mathrm{t} 1) \mathrm{W}(\mathrm{t} 2)]=\min (\mathrm{t} 1, \mathrm{t} 2)$.

\section{Model Parameter Estimation}

After arranging for the collected test data, we get the bearing relative temperature $\mathrm{Xi}(\mathrm{t})$ for the ith test sample at time $t j$, then we take points from the above bearing relative temperature data as follows: (t0, Xi(t0)), (t1, Xi(t1)), ..., (tk, Xi(tk)), and $\mathrm{t} 0 \leq \mathrm{t} 1 \leq \ldots$ $\leq \mathrm{tk} \leq \mathrm{tm}$. Noting that $\mathrm{Xi}=[\mathrm{Xi}(\mathrm{t} 0), \mathrm{Xi}(\mathrm{t} 1), \ldots, \mathrm{Xi}(\mathrm{tk})]$. By Eq. (1), for the ith test sample, we have

$$
\Delta \mathrm{xij}=\mathrm{vi} \Delta \mathrm{tj}+\delta \mathrm{i} \Delta \mathrm{W}(\mathrm{tj}) .
$$

Where $\Delta \mathrm{xij}=\mathrm{Xi}(\mathrm{tj})-\mathrm{Xi}(\mathrm{tj}-1), \Delta \mathrm{tj}=\mathrm{tj}-\mathrm{tj}-1, \Delta \mathrm{W}(\mathrm{tj})=\mathrm{W}(\mathrm{tj})$ - W(tj-1), i=1, 2, ..., n, j=1, 2, .., k.

According to the definition of Wiener process, we can know $\Delta \mathrm{W}(\mathrm{tj}) \sim \mathrm{N}(0, \Delta \mathrm{tj})$, thus we have

$$
\Delta \mathrm{xij} \sim \mathrm{N}(\mathrm{vi} \Delta \mathrm{tj}, \delta \mathrm{i} 2 \Delta \mathrm{tj})
$$

The Wiener process has the property of stationary independent increments, so we can get the joint PDF about $\Delta x i 1, \Delta x i 2, \ldots, \Delta x i k$, i.e. the sample likelihood function is: 


$$
\mathrm{L}(\mathrm{vi}, \delta \mathrm{i})=\mathrm{f}(\Delta \mathrm{xi} 1, \Delta \mathrm{xi} 2, \ldots, \Delta \mathrm{xik})=\mathrm{f}(\Delta \mathrm{xi} 1) \mathrm{f}(\Delta \mathrm{xi} 2) \ldots \mathrm{f}(\Delta \mathrm{xik})
$$

Substituting (3) in (4), and taking logarithm on both sides of the equal sign in (4), we get

$$
\begin{aligned}
& \operatorname{lnL}\left(v_{i}, \delta_{i}\right)=\ln \prod_{j=1}^{k} f\left(\Delta t_{j}\right)= \\
& \sum_{j=1}^{k}\left(\ln \frac{1}{\sqrt{2 \pi \delta_{i}^{2} \Delta t_{j}}}-\frac{\left(\Delta x_{i j}-v_{i} \Delta t_{j}\right)^{2}}{2 \delta_{i}^{2} \Delta t_{j}}\right)
\end{aligned}
$$

Then calculating separately the partial derivative to vi and $\delta i$ in (5), we get

$$
\begin{gathered}
\frac{\partial \ln \mathrm{L}}{\partial \mathrm{v}_{\mathrm{i}}}=\sum_{\mathrm{j}=1}^{\mathrm{k}} \frac{\Delta \mathrm{x}_{\mathrm{ij}}-\mathrm{v}_{\mathrm{i}} \Delta \mathrm{t}_{\mathrm{i}}}{\delta_{\mathrm{i}}^{2} \Delta \mathrm{t}_{\mathrm{j}}}= \\
\frac{\partial \ln \mathrm{L}}{\partial \delta_{\mathrm{i}}}=\sum_{\mathrm{j}=1}^{\mathrm{k}}\left(-\frac{1}{\delta_{\mathrm{i}}}+\frac{\left(\Delta \mathrm{x}_{\mathrm{ij}}-\mathrm{v}_{\mathrm{i}} \Delta \mathrm{t}_{\mathrm{j}}\right)^{2}}{\delta_{\mathrm{i}}^{3} \Delta \mathrm{t}_{\mathrm{j}}}\right)=0
\end{gathered}
$$

Thus, through solving the equations consisting of (6) and (7), we get the maximum likelihood estimate of $v_{i}$ and $\delta_{i}$ as follows:

$$
\begin{aligned}
& \hat{\mathrm{v}}_{\mathrm{i}}=\frac{1}{\mathrm{k}} \sum_{\mathrm{j}=1}^{\mathrm{k}} \frac{\Delta \mathrm{x}_{\mathrm{ij}}}{\Delta \mathrm{t}_{\mathrm{j}}} \\
& \widehat{\mathrm{\delta}}_{\mathrm{i}}=\left[\frac{1}{\mathrm{k}} \sum_{\mathrm{j}=1}^{\mathrm{k}} \frac{\left(\Delta \mathrm{x}_{\mathrm{ij}}-\hat{\mathrm{v}}_{\mathrm{i}} \Delta \mathrm{t}_{\mathrm{j}}\right)^{2}}{\Delta \mathrm{t}_{\mathrm{j}}}\right]^{\frac{1}{2}}
\end{aligned}
$$

Assuming the sample size is $\mathrm{n}$, we can get every test sample of drifting coefficient $\mathrm{D}_{\mathrm{v}}=\left\{\hat{\mathrm{v}}_{1}, \widehat{\mathrm{v}}_{2}, \cdots, \hat{\mathrm{v}}_{\mathrm{n}}\right\}$ and diffusion coefficient $D_{\delta}=\left\{\hat{\delta}_{1}, \widehat{\delta}_{2}, \cdots, \widehat{\delta}_{n}\right\}$ and noting the original value of bearing relative temperature is $\mathrm{D}_{\mathrm{b}}=\left\{\mathrm{b}_{1}, \mathrm{~b}_{2}, \ldots, \mathrm{b}_{\mathrm{n}}\right\}$ Where $\widehat{\mathrm{v}}_{\mathrm{i}}$ and $\widehat{\delta}_{\mathrm{i}}$ is respective the estimating value of drifting coefficient and diffusion coefficient of the ith bearing, bi is the original temperature of the ith bearing, $i=1$, $2, \ldots, n$.

\section{Example Analysis}

Due to the HSTB test is still in the design stage, it is unable to provide the required test data for verifying the validity of the above method.

Consequently, we use the test data of two bearings, which is similar to the HSTB. By the data, we know that the initial value of bearing relative temperature is respective $b(1)=-0.2^{\circ} \mathrm{C}$ and $b(2)=-$ $0.9^{\circ} \mathrm{C}$. After extracting the data points from the test data every minute and removing the abnormal data, we get the 245 degradation data points shown in Fig. 2. For the HSTB, considering it is hard to get good data in a short time since the reliability requirement is higher. The degradation rate is smaller and the degradation process is also slower, and the design index requirement is expect for a year for the bench test. Therefore, we can extend appropriately the interval time of extracting data points to obtain effective performance degradation data.

Assuming that performance degradation data measured at any moment obey the normal distribution, according to the virtually expanded sample method, we can augment virtually the size of test sample from $n=2$ to $n=10$. Thus, we get the initial moment expanded samples as:

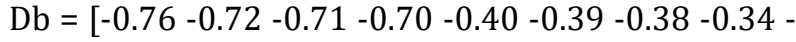
$0.2-0.9]$.

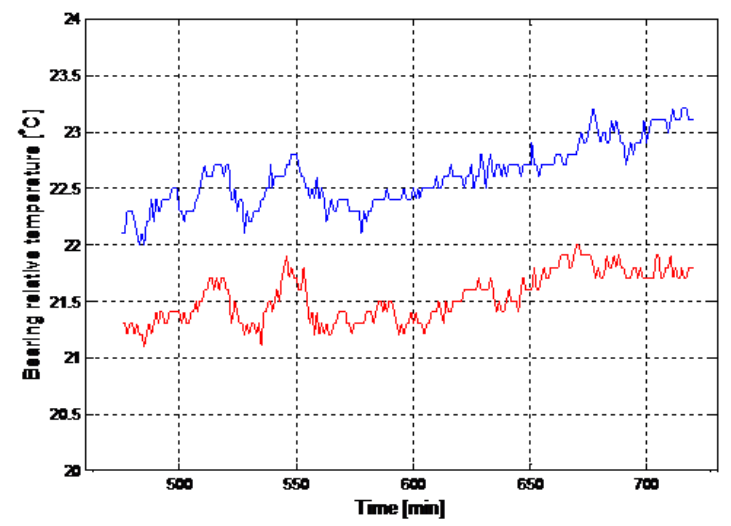

Figure 2: Processed bearing performance degradation data

Simultaneously, for the processed test data measured at other moment, the corresponding expanded sample data is shown in Fig. 3.

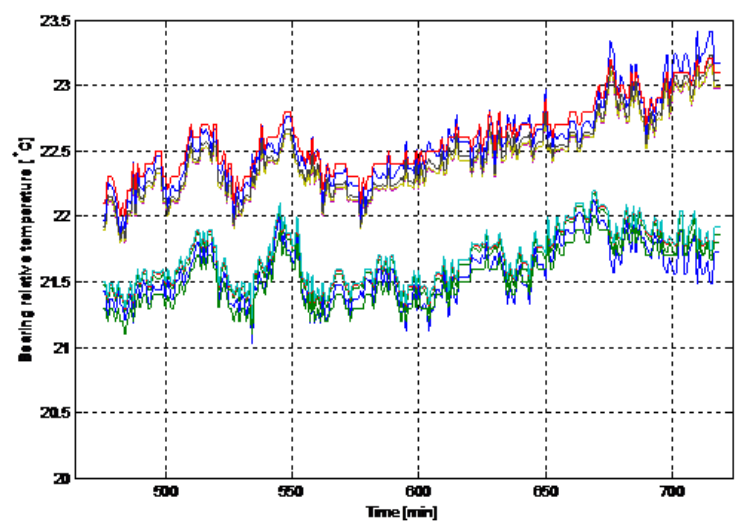

Figure 3: Expanded bearing performance degradation data

After getting the virtually expanded sample, the drifting coefficient $\mathrm{v}$ and diffusion coefficient $\delta$ can be calculated, thus we get their estimation values are respective: 


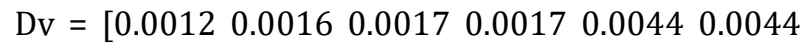
$0.00460 .00490 .00200 .0041]$,

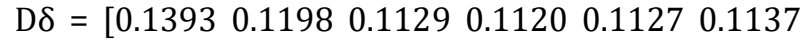
$0.12060 .14010 .09380 .0953]$.

Using the Bootstrap estimation method, we get 1000 sets of Bootstrap sample, through calculation we have:

$$
\begin{aligned}
& \tilde{\mu} b=-0.553, \tilde{\delta} 2 b=0.097 ; \\
& \tilde{\mu} v=-0.003, \tilde{\delta} 2 \mathrm{v}=2.27 \times 10-6 .
\end{aligned}
$$

Then averaging for the diffusion coefficients of bearings expanded, we get:

$$
\tilde{\delta}=(\hat{\delta}(1)+\hat{\delta}(1)+\ldots+\hat{\delta}(10)) / 10=0.116 .
$$

As the requirement of bearing design index, the bearing is regarded as failure when the bearing temperature is 40 degrees more than the ambient temperature. That is the failure threshold of bearing relative temperature is 40 degrees. So substituting the estimate of above parameters and the failure threshold in Eq. (5) and (6), we get the distribution function and PDF and reliability function of bearing life shown in Fig.4 - Fig. 6.

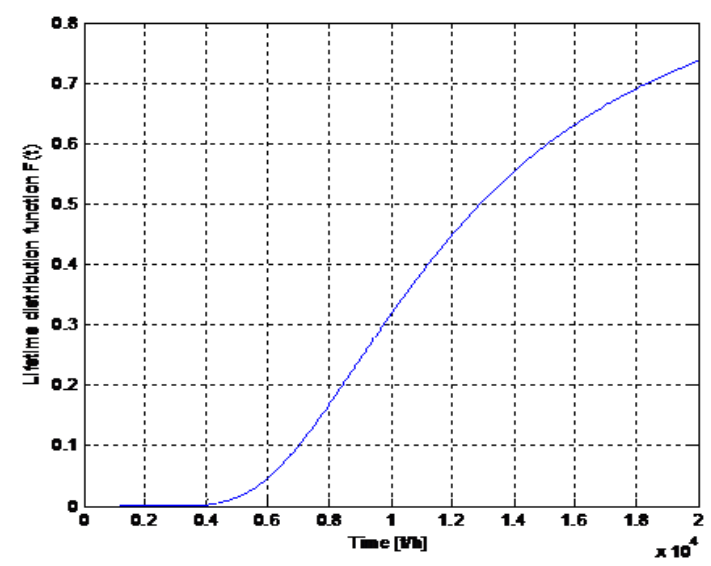

Figure 4: Distribution function of bearing life

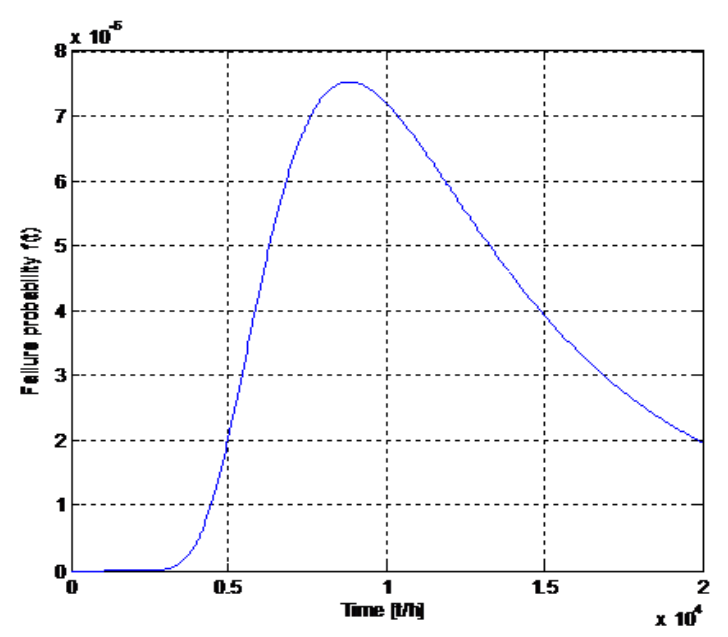

Figure 5: PDF of bearing life

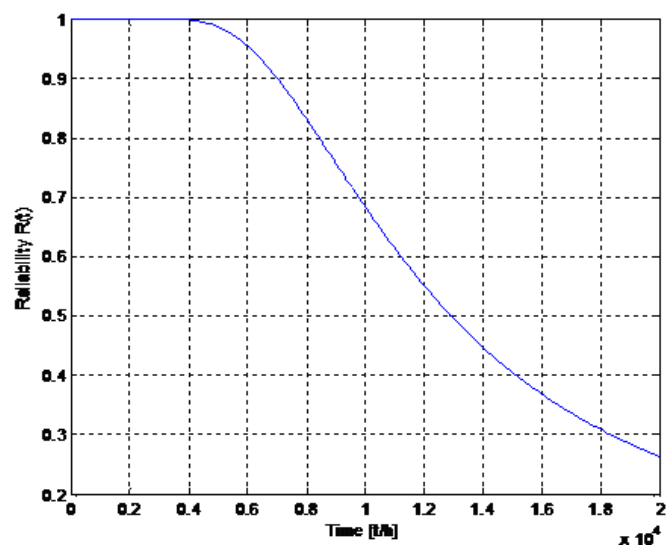

Figure 6: Reliability function of bearing life

In Fig. 6, we can clearly observe that the reliability of test bearings is very high, almost $100 \%$, within 4000 h. At the same time, after the bearing works for 6000 $h$, with the continual wear and tear of bearing operation, the trend of reliability decline is gradually increase. Combined with the Figs. 4-6, we calculate to get that the time of bearing working is $4825 \mathrm{~h}$ when its reliability is $99 \%$. This result meets basically the request of the tested bearings, which are installed in the train which its highest speed is 200 kilometers per hour. But considering that the observation data is only recorded for $12 \mathrm{~h}$, and it is not enough to evaluate the high reliability products, so that there is a little gap between the above gained result and the information provided by the manufacturer. Therefore, in order to better evaluate the bearing reliability life, we still need long test time to make the necessary correction for the result of reliability evaluation.

\section{Conclusion}

Since it is difficult to get the failure data for HSTB test, the performance degradation can be regarded as an important source of information for reliability evaluation. Compared with the traditional method based on the data of life failure, the performance degradation data can be real-time measured in the period of the bearing operation, and the reliability evaluation result can be further modified with the running of test, so it is helpful to get more sufficient reliability information in the case of not adding additional test.

Meanwhile, for the products of batch production, the differences of product performance may be caused by the differences in processing technology, manufacturing error and assembling technology.

Therefore, in order to forecast the different products life and know the overall characteristic, we take the unknown coefficients in the model as random variables, so that it can well consider the 
differences between individuals and avoid reducing the accuracy of reliability assessment.

Future work may aim to study multiple performance degradation indexes to improve the efficiency of reliability assessment, because it needs generally longer test time in only single performance index.

\section{References}

[1]Zhu De-xin, Liu Hong-zhao. Reliability evaluation of high-speed train bearing with minimum sample [J]. Journal of Central South University (Science and Technology), 2013, 44(3): 963-969.

[2]Zhu De-xin, Liu Hong-zhao, Yuan Da-ning, et al. Time determination and life assessment of highspeed railway bearing reliability test [J]. China Mechanical Engineering, 2014, 25(21): 28862891.

[3]Danish M Y, Aslam M. Bayesian in ference for the randomly censored Weibull distribution [J]. Journal of Statistical Computation and Simulation, 2014, 84(1): 215-230.

[4]Balka J, Desmond AF, McNicholas PD. Review and implementation of cure models based on first hitting times for Wiener Processes [J]. Lifetime Data Analysis, 2009, 15(12): 147-176.
[5]Tsai C C,Tseng S T,Balakrishnan, N.Optimal design for degradation tests based on Gamma processes with random effects[J]. IEEE Transactions on Reliability, 2012, 61(2): 604613.

[6]Jiang Xi, Liu Hong-zhao, Zi Jiao-jiao, et al. Extremely small sample's reliability of motorized spindle based on Bayes method[J]. Journal of Vibration and Shock, 2015, 34(4): 121-127.

[7]Yang Ren. Improved MML method of system reliability synthesis based on Bayesian theory [J]. Journal of Sichuan Ordnance, 2015(9) :158-161.

[8]Si Xiao-sheng, Wang Wen-bin, Hu Chang-hua, et al. A Wiener-process-based degradation model with a recursive filter algorithm for remaining useful life estimation [J]. Mechanical Systems Signal Processing, 2013, 35(1-2): 219-237.

[9]Tseng S T, Tang J, Ku I H. Determination of optimal burn-in parameters and residual life for highly reliable products[J]. Naval Research Logistics, 2003, 50: 1-14.

[10]Tang J, Su TS. Estimating failure time distribution and its parameters based on intermediate data from a Wiener degradation model [J]. Naval Research Logistics, 2008, 55(3): 265-276. 